\title{
Chinese Residency and Fellowship Training: Quo vadis?
}

Xin Chen ${ }^{1}$, Liang Gao ${ }^{2, *}$, Hu Zhang ${ }^{3}$, Hong Chai ${ }^{4}$

1Department of Urology, The Second Affiliated Hospital of Anhui Medical University, Hefei, China

${ }^{2}$ Institute of Experimental Orthopaedics and Osteoarthritis Research, Saarland University, Homburg/Saar, Germany

${ }^{3}$ Department of Obstetrics and Gynecology, Shanghai Pudong Hospital, Fudan University Pudong Medical Center, Shanghai, China

${ }^{4}$ Department of Nuclear Medicine, Shanghai Jiao Tong University Affiliated Sixth People's Hospital (East Campus), Shanghai, China

${ }^{*}$ Corresponding to:

Liang Gao, MD, Institute of Experimental Orthopaedics and Osteoarthritis Research, Saarland University, Kirrberger Street 100, Building 37, Homburg/Saar, 66421, Germany. liang.gao@uni-saarland.de 
As a crucial part of the China's healthcare reform, the Chinese Standardized Residency Training Program was released in mainland China in $2013^{1}$. At the end of 2014, 55000 resident physicians had been trained in 8500 residency programs from 559 hospitals across the country. In 2016, the national government initiated the Chinese Subspecialty Fellowship Training Program (CSFTP) with the aim to establish an initial framework of the training by 2020. On June 12, 2017, the Chinese Medical Doctor Association, directly affiliated with the National Health and Family Planning Commission of China, officially announced the first group of hospitals for the pilot phase of CSFTP in three subspecialties, including neurosurgery, respiratory and critical care medicine, and cardiovascular disease ${ }^{2}$ (Figure 1A).

Altogether, the pilot phase of CSFTP initiates with 54 fellowship programs in neurosurgery, 79 in respiratory and critical care medicine, and 69 in cardiovascular medicine. A total of 106 hospitals from mainland China were involved (Figure 1B). These included hospitals are mainly large tertiary hospitals or academic medical centers located in the economically developed regions of mainland China (e.g. 14 hospitals in Beijing, 14 in Shanghai, and 8 in Guangdong).

Similar to the American counterpart, for Chinese medical residents, to participate a fellowship training program will also be voluntary on a highly completive basis. The length of fellowship training may vary in different subspecialties and will require an additional two to four years beyond the standard three-year general residency training.

Yet, compared to their American peers, the Chinese young doctors are facing two distinctive challenges under the current health care system. The first one is the working environment. Trainees in the tertiary hospitals of China are suffering from a stressful workload with unsatisfied salary and sometimes even under physical or verbal violence ${ }^{3}$. Of note, patient-physician conflicts also force supervisors to be reluctant to allow trainees to perform patient care independently ${ }^{4}$. The other 
challenge is the program organization ${ }^{5}$. The training process, varying from hospital to hospital, is predominantly tutor-centered and passive learning for the trainees. Journal clubs with cutting edge literature and formal "hands-on" technique workshops are inadequately provided.

To sum, formal residency and fellowship training has become a worldwide necessity for the medical profession, China has now followed this path. There is a lot to be learnt and improved before both Chinese residency and fellowship training is truly "standardized and competent".

We declare no competing interests. 


\section{References}

1. National Health and Family Planning Commission of China. The instructions on pilot implementaiton of residuency standardization training.

http://www.nhfpc.gov.cn/qjjys/s3593/201401/032c8cdf2eb64a369cca4f9b76e8b059.s html. (accessed June 17, 2017; in Chinese).

2. Chinese Medical Doctor Association. The notice of the first groups of hospitals of the pilot phase of the Chinese Subspecialty Fellowship Training Programs. http://www.cmda.net/xiehuixiangmu/biyehouyixuejiaoyubu/tongzhigonggao/2017-0612/15646.html. (accessed June 17, 2017; in Chinese).

3. Wu LX, Qi L, Li Y. Challenges faced by young Chinese doctors. The Lancet 2016; 387(10028): 1617.

4. Yueju L. Violence against doctors in China. The Lancet 2014; 384(9945): 745.

5. Lio J, Dong H, Ye Y, Cooper B, Reddy S, Sherer R. Standardized residency programs in China: perspectives on training quality. International Journal of Medical Education 2016; 7: 220. 
Figure 1. Overview of the pilot phase of the Chinese Subspecialty Fellowship Training Program in mainland China. (A) Geographical distribution of the fellowship training programs in the three subspecialties (neurosurgery, respiratory and critical care medicine, and cardiovascular medicine). (B) Bar chart of number of hospitals involved from different provinces (municipalities and autonomous regions).

A

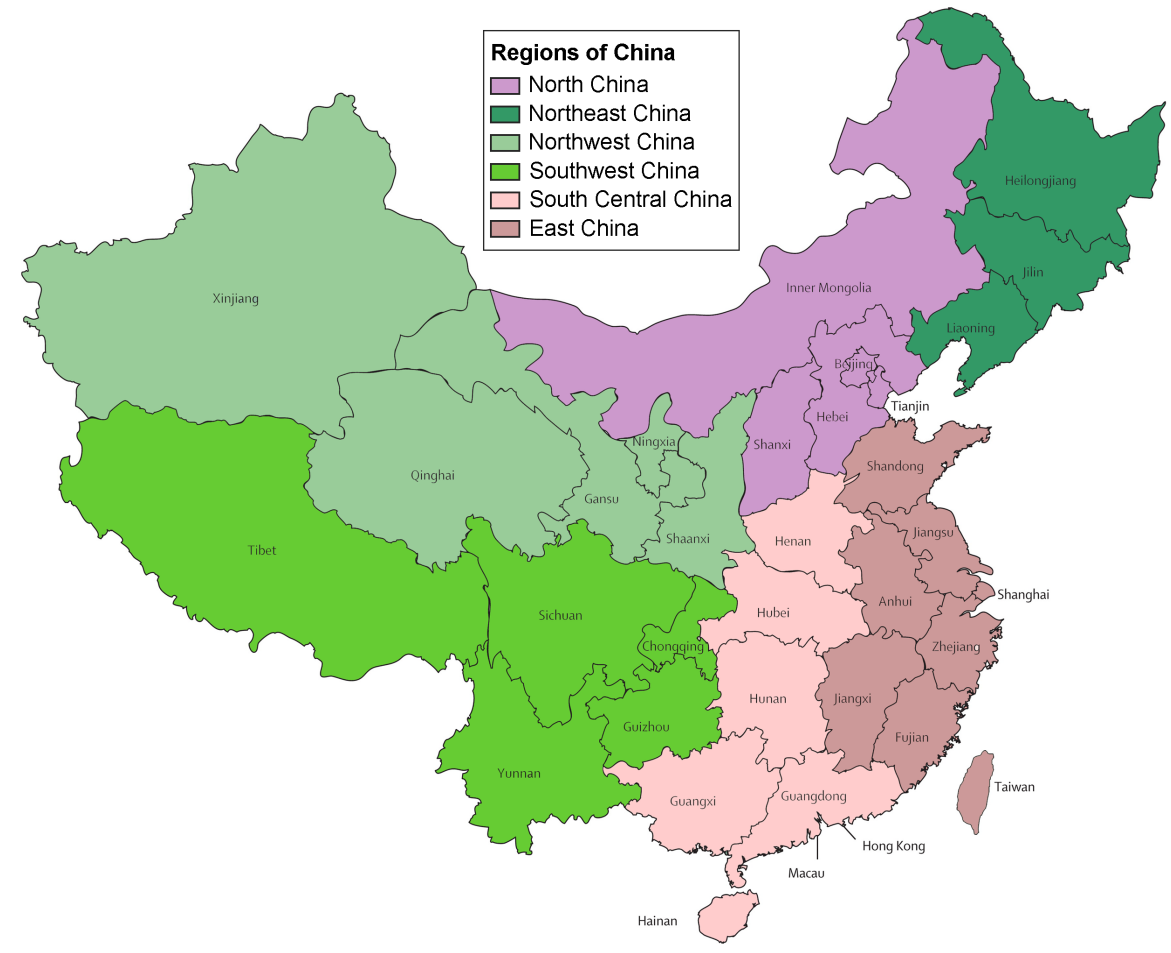

\begin{tabular}{lccc} 
Regions of China & Neurosurgery & $\begin{array}{c}\text { Respiratory and } \\
\text { Critical Care Medicine }\end{array}$ & Cardiovascular Medicine \\
\hline$\square$ North China & 10 & 16 & 15 \\
$\square$ Northeast China & 6 & 8 & 7 \\
$\square$ Northwest China & 4 & 6 & 5 \\
$\square$ Southwest China & 4 & 10 & 5 \\
$\square$ South Central China & 10 & 14 & 13 \\
$\square$ East China & 20 & 25 & 24 \\
\hline Total number of programs* & 54 & 79 & 69 \\
\hline
\end{tabular}

*Without Hongkong, Macau, and Taiwan.

B

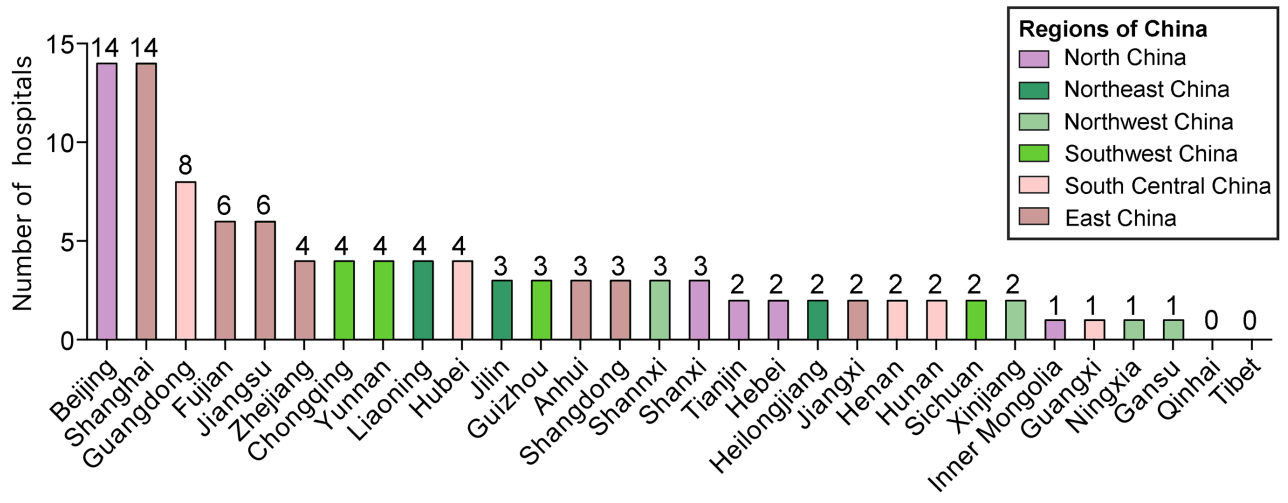

